\title{
Smart factory and education: an integrated automation concept
}

\section{Marcus Kurth*, Carsten Schleyer and Daniel Feuser}

Faculty of Mechanical Engineering, University of Applied Sciences,

Constance, Germany

Email: Marcus.Kurth@htwg-konstanz.de

Email: carsten.schleyer@htwg-konstanz.de

Email: daniel.feuser@htwg-konstanz.de

*Corresponding author

\begin{abstract}
The introduction of cyber physical systems into production companies is highly changing working conditions and processes as well as business models. In practice, a growing discrepancy between big and small respectively medium-sized companies can be observed. Bridging that gap, a university smart factory is introduced to give that companies a platform to trial, educate employees and access consultancy. Realising the smart factory a highly integrated, open and standardised automation concept is shown comprising single devices, production lines up to a higher automation system maintaining a community or business models.
\end{abstract}

Keywords: cyber physical systems; CPSs; smart factory; education; internet of things; data integration and visualisation.

Reference to this paper should be made as follows: Kurth, M., Schleyer, C. and Feuser, D. (2017) 'Smart factory and education: an integrated automation concept', Int. J. Service and Computing Oriented Manufacturing, Vol. 3, No. 1, pp.43-53.

Biographical notes: Marcus Kurth is a Professor for Control and System Engineering at the University of Applied Sciences in Constance (HTWG) since 2011. He is a member of the Institute for System Dynamics and Head of the Demonstration Factory (Modellfabrik) Lake of Constance. Before, he was with Evonik Energy Services GmbH as Head of advanced process control.

Carsten Schleyer studied Industrial Engineering at Technical University of Darmstadt, Germany and Technical University of Linkoeping, Sweden and a $\mathrm{PhD}$ at RWTH Aachen, Germany. Then, he has started his business career and was working for international companies in the field of supply chain and production. Since October 2011, he is a Professor at the Hochschule Konstanz University of Applied Sciences (HTWG). His research activities are in the field of Lean Management, Smart Manufacturing, and Industry 4.0. Since 2015, he is the Head of the Model Factory Industry 4.0 (www.modellfabrikbodensee.de) at Hochschule Konstanz University of Applied Sciences (HTWG).

Daniel Feuser is a Research Associate at the Modellfabrik Bodensee (Faculty of Mechanical Engineering, HTWG Constance). He studied Electrical Engineering and Management at the University of Applied Sciences in Constance, Germany. 
This paper is a revised and expanded version of a paper entitled 'Smart factory and education - an integrated automation concept', presented at 11th IEEE ICIEA, Hefei, China, 5-7 June 2016.

\section{Introduction}

The integration of humans, things and machines grows rapidly. Digitisation and automation lead in capital investment markets to faster product cycles and a globally rising competitive pressure. The term cyber physical system (CPS) describes the digitisation and improved flexibility of production lines. Introducing a smart production line allows the fabrication of products with a high degree of individualisation. For that production lines, machines and products respectively semi-finished products should be linked (Hesse et al., 2014). The low degree of automation in production lines compared to continuous systems can be raised highly leading to more efficient production and logistic processes by networking. Goal of introducing CPSs is to link automation components, processes and products over a network in form of cloud computing. CPS interact with sensors, actuators and data processing smart, largely autonomous, decentral and communicate often via wireless communication networks (VDI, 2014). Condition monitoring allows to control and analyse states as well as production and process data to derive needs for action and operation recommendations (Hesse et al., 2014; ACATECH, 2013).

However, investigations show that only a quarter of companies have the basis for CPS, i.e., sufficient linked machines, in operation already (Spath et al., 2014). About $16 \%$ do not even know the term CPS and only $4 \%$ produce products with CPS (Techconsult, 2014). Especially the industrial small and medium-sized businesses fear the high kick-off investments and the uncertainty of the cost-effectiveness (Rogers, 2003). Practice-oriented test and laboratory facilities are missing to test and familiarise new business model in a company own context and the transformation process of the company is highly complex. Those companies face a high uncertainty according the effort required for the data acquisition and processing as well as for the data and process security.

Stimulating the success of CPS and its technology especially for small and middle-sized companies a laboratory and testing facility is operated at the University of Applied Sciences in Constance for trial, education and consultancy. The factory is financed by the government and industrial partners. The production lines follow the lean production concepts which are transferred from the material to the energy flow up to the information processed in a highly integrated automation concept. The applied network concept builds up on internet principles - but considering the requirements like real-time processing and big data of a shop floor.

In an actual research project a third production line is build up as a decentralised, cross border production line in cooperation with two other universities around the Lake of Constance in Germany, Austria and Switzerland together with a logistic partner. The goal is to bring complete transparency into the supply change preventing system dynamic effects like the bullwhip effect. 


\section{Educational concept}

In view of the rapidly advancing digitisation, not only in everyday life, but especially in the industrial production process across the entire value chain, there are unavoidable needs to deal with technological as well as economic and socially relevant questions. The smart factory of the HTWG Constance, as shown in Figure 1, meets the requirements in the three key areas of teaching, research and knowledge transfer.

The objective of the smart factory is to achieve and to illustrate an optimised production. Problems and questions rising up here are exactly the real challenges of companies: Which technologies should be used? How can the necessary components be procured, in order to be able to build up an optimal automation system? What about the relationship between production possibilities, customer requirements, delivery conditions, logistics, etc.?

Figure 1 Smart factory of Htwg Constance (see online version for colours)
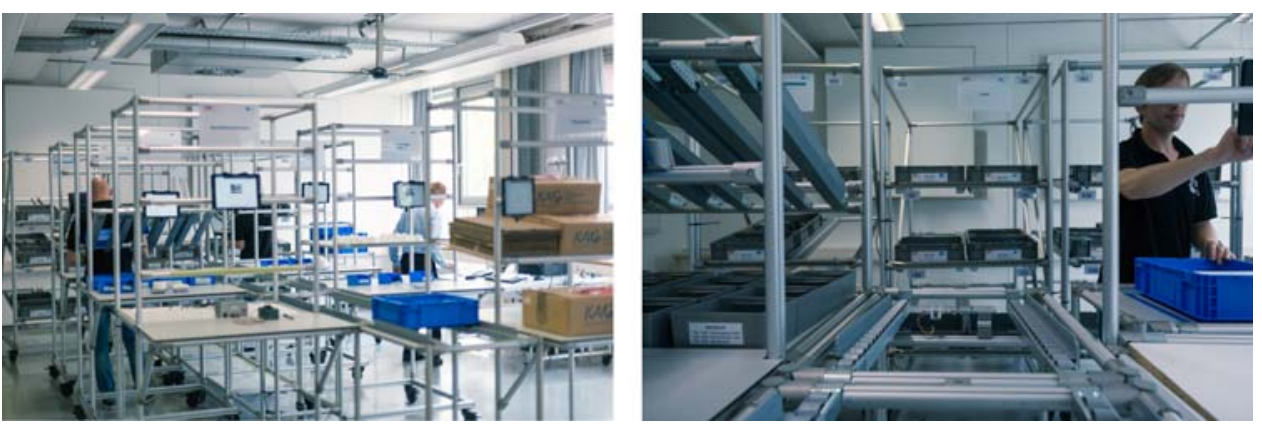

The smart factory as a laboratory and testing facility should allow to learn and understand the operation modes of modern production as well as the single components of it like machines, work stations and cyber physical products. That contains aspects of machine communication, programming, processing and assessment of data aiming on an optimal state of operation of the production line and products adopted - work flow. Focussing on a high standardisation, self-optimisation and organisation the degree of automation is stepwise increased and with that efficiency and productivity.

To keep the holistic systemic approach that thought affect not only the production line. Furthermore, the concept is adopted to products, organisation and business models up to the education of the employees.

The developed concept targets schools, colleges, universities and companies with training on the job. Working on real and industry-oriented products, students and employees of companies, using the smart factory as training facility, learn something about the context of digitisation and the processes of a digitised production.

The schools respectively colleges cover the cyber physical components and products. Used is a cyber physical product with a high functionality, i.e., programmable, data acquisition, networking, smart and remote controllable. According the high degree of standardisation that product represents also a component of the production line in the smart factory at the same time. With that it is possible to teach and understand all requirements on modern cyber physical machines. 
The university in that concept covers the production line and smart factory. The cyber physical product introduced before is actually produced there. That also comprises customer individual products, interconnecting machines or work places and interaction with design and organisation methods like lean production, energy efficiency or ergonomics.

Side effects are that students are attracted to STEM fields and employees faced with the structural changes can be trained in the sense of a lifelong learning accompanied by the universities. Furthermore, researchers from different disciplines, who have no access to real objects such as production data, can find a test environment. At the same time, the smart factory is a demonstration environment on the campus.

\section{The cyber physical product}

The CPS produced exemplary in the smart factory is a smart vehicle with a modular design, as shown in Figure 2. Basis is a vehicle chassis build up with wheels, base-plate to attach an automation system on it and two motors. The motors can be used to drive the car both or as an alternative one for driving and one for steering. Batteries are used for energy supply. For interaction with the surroundings of the vehicle several sensors including distance sensors and a camera can be connected.

Figure 2 Smart vehicle as cyber physical product (see online version for colours)

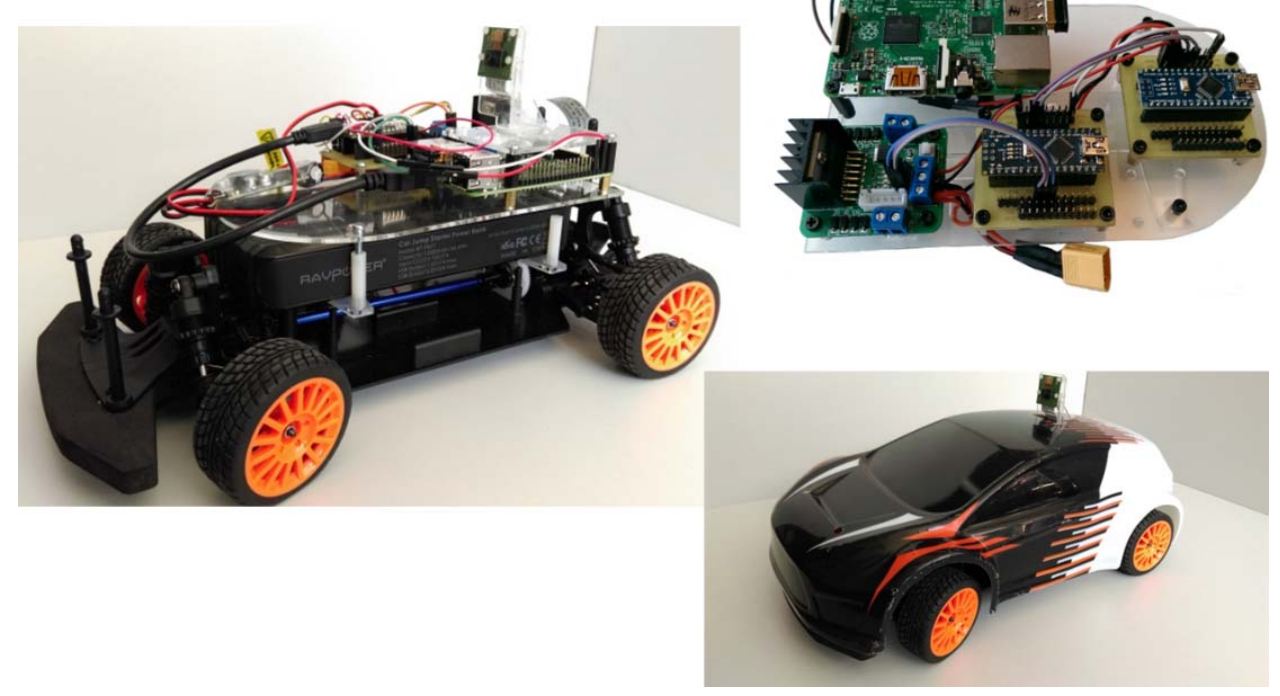

The vehicle is able to communicate to other vehicles, a supervising level or with the human machine interface (HMI) via a modified WLAN or radio communication. With that a first person video can be streamed as well as control signals can be send to the vehicle in real-time. As HMI each device equipped with a standard WLAN module can be used, e.g., a smart phone together with a web browser. The browser window shows the video stream and sensor data, control is performed by touch screen, mouse or keyboard depending on the used device. 
The CPS is built hierarchically applying the automatisation pattern as shown in Figure 3. The HMI is the highest level layer. Information can be sent into it and being displayed by the system. An example for the output of information is a real-time video stream from the camera. The camera is used for first person video streaming as well as for object recognition. The HMI is connected as a client to a Raspberry Pi (RPI) via Wifi, which is acting as a server and having control over the system. It is communicating with the camera via CSI-bus and delivers analogue audio data as well.

Figure 3 Automation scheme of the smart vehicle (see online version for colours)

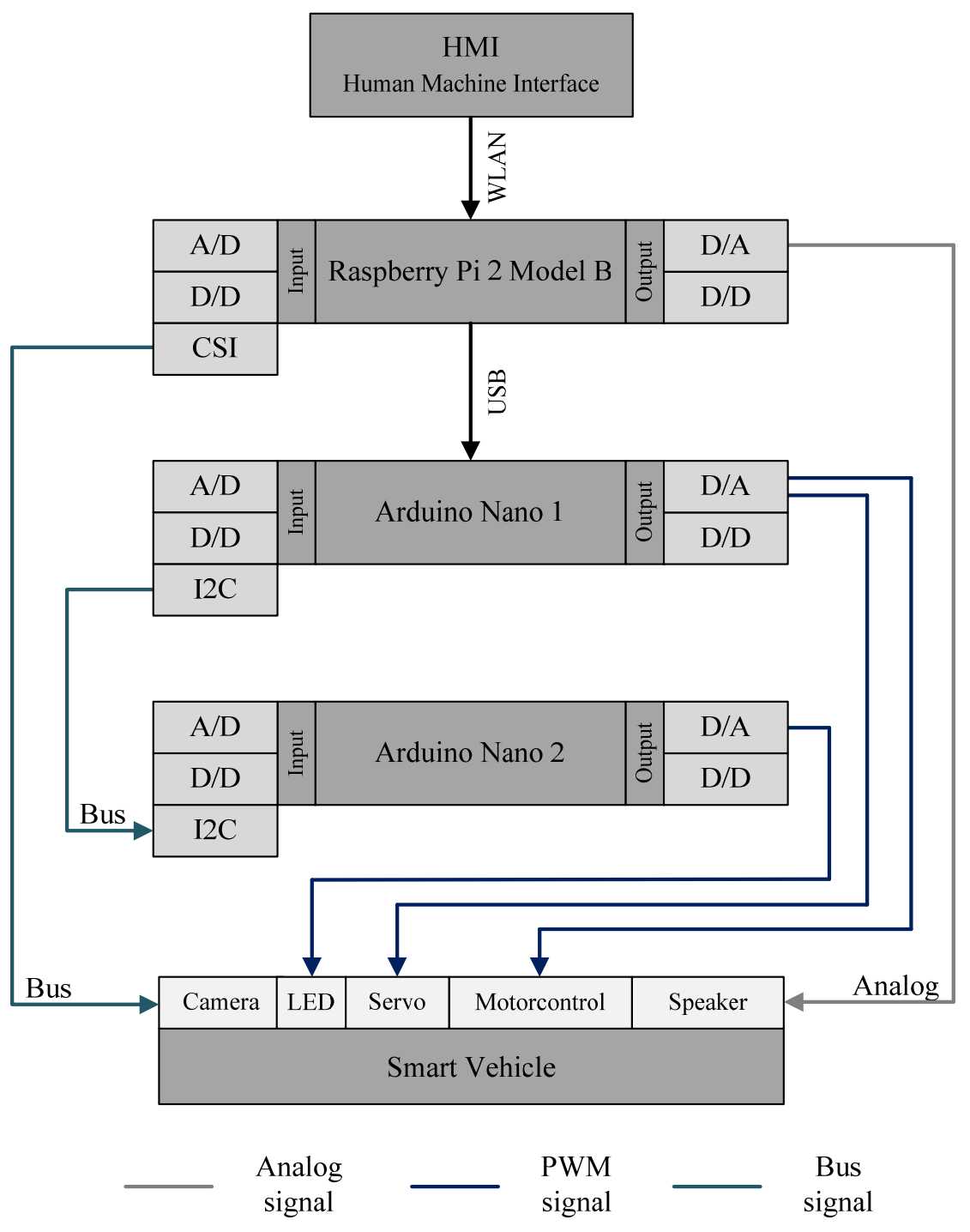

The CPS is completed with two Arduino Nano Microcontrollers, which are triggering the actors (servomotors, a motor control and two LED modules) via pulse width modulation (PWM). The hardware resources of an Arduino Nano are restricted. Therefore, just one 
microcontroller is not sufficient to control all built in hardware components. The RPI and the first Arduino Nano are linked together by a serial USB interface. This bi-directional connection is used for data transmission. The control data is provided to the second microcontroller via $\mathrm{I} 2 \mathrm{C}$-bus.

The microprocessor-based automation system can be programmed in a high level programming language or preferable graphically, latter allowing the implementation of complex algorithms easily app oriented. However, of course, the vehicle can be operated alternatively by classical electronic components like logic devices or op-amps.

The radio communication is optional and used where a longer distance connection is required, in that case here a supervising drone coordinating ground vehicles.

The concept behind that CPS product is a low budget embedded system and offers the possibility to become familiar with all aspects and functionality required by modern machines in a smart production line.

Using modern manufacturing methods like 3D printing or laser cutting building the parts of the vehicle that product can highly be individualised by the same manufacturing methods according the costumers wishes later in the series production of the smart factory.

\section{The smart factory}

Within the smart factory, the above describes CPS is produced under demonstration of CPS components. The production line represents basically a typical plant with a conventional control and a comparable low degree of automation representing the actual state of real production lines in many companies. The production then can be leveled up stepwise by CPS elements like traceability, energy consumption, OEE analysis, etc. With that the degree of automation is stepwise raised and the work places with manual work are gradually integrated into the automation system. That stepwise raising of the degree of automation during the education of students documents the particular progress by the used technique and the interaction of the students.

Figure 4 shows the layout of the smart factory of the HTWG Constance. Order specific parts are automatically picked out of the high-bay rack and transported by an automated guided vehicle (AGV) or robot to the fully automated production line or to the work places with manual labour. The fully automated production line produces circuit boards for the PWM and sensor boards according the order specific requirements.

At the end of that production line, an industrial robot picks the circuit boards and hands them over to a hybrid assembly line build up out of three work places. On that digitalised and networked work places one to three workers assemble parts of the CPS product supported by an automated guiding system. The work places are optimised according to ergonomically as well as to lean aspects. Further, according the connection to the WLAN on the IT side and to the robot respectively the AGV to the material side this places build the interface to the HMI.

Another robot delivers at that stage the customer individual parts for customer specific assembly of the product. A real CPS product has emerged.

The customer individually designed parts are freely designed components like spoilers, rims, etc., - created by the customer himself with the help of specifically guided apps. Those parts are produced on a 3D-printer or laser cutters. 
After the work places the quality check follows and the vehicles have to perform on a test track. If errors are detected according the customised specification a robot brings the affected product to a rework place.

A successful quality check leads the product to the packaging and shipping stage.

The complete production process of each product is documented to achieve traceability. Data of machines, production line and work places is processed in a realtime factory cloud solution which is also used for inter order communication of the single stages. The used protocol was developed by the university applying modified standard protocols. Non-modified devices using standard protocols can be integrated as well. From a didactic point of view, standard bus protocols as well as manufacturer specific real-time protocols up to programming of the university own protocol can be shown.

The factory cloud controls orders and order specific information is reported. The single autonomous systems like robots or AGV and the networked work space including the automated production line and high-bay stack are able to communicate in such a way that the material flow through the factory is fully organised by that decentral automation system.

Figure 4 Layout of the smart factory of HTWG Constance (see online version for colours)
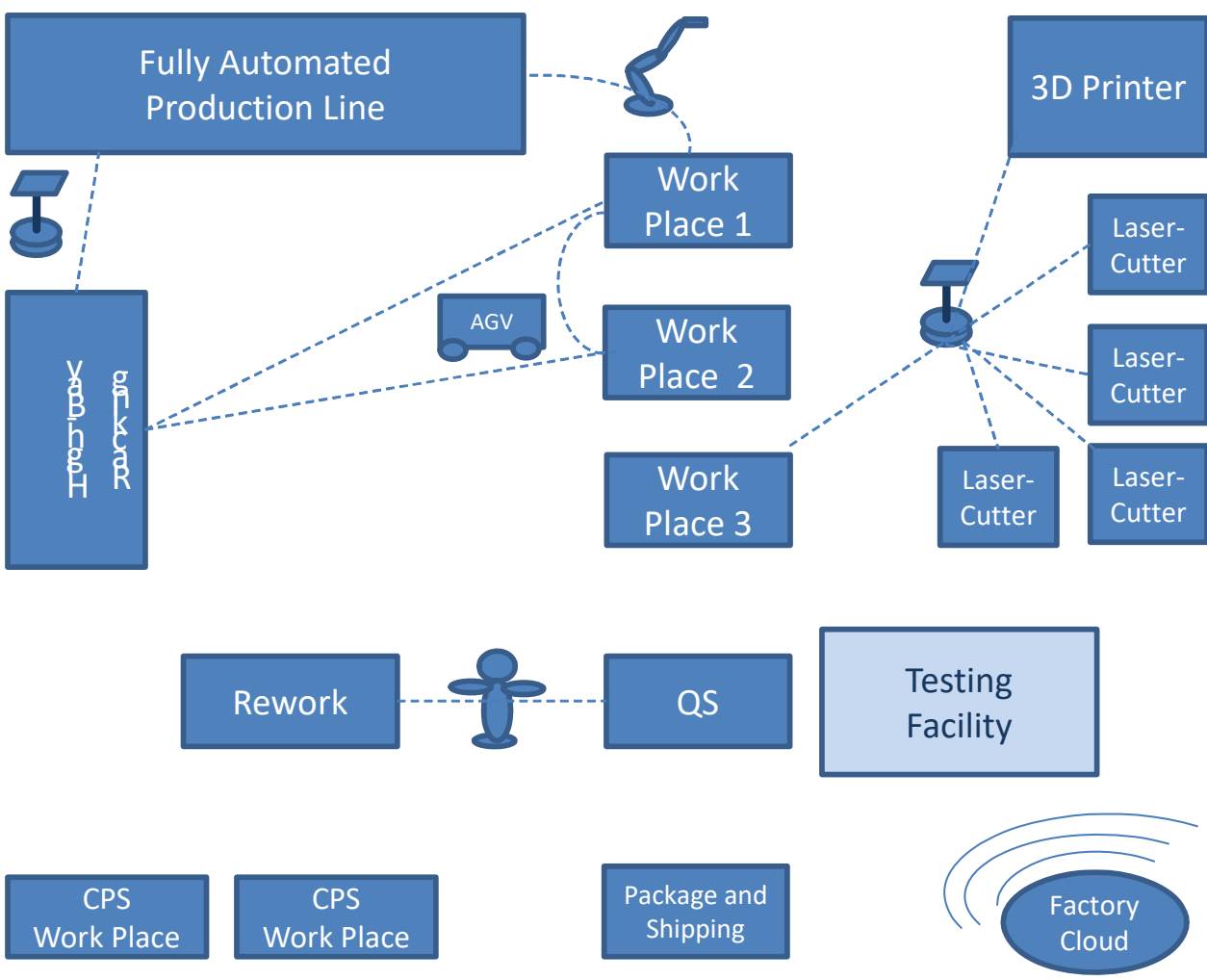

The customers' orders communicate via the factory cloud with the actually concerned stages and determine how a stage has to process the product. At the same time, the process is fully documented. Further actual machine data is logged and assessed. 
The smart factory allows several customer individualisation of the CPS product where the customer becomes his own designer together with the already foreseen several products variants which a customer can order.

The product variants are options provided by the manufacturer and which the customer can check during the order process like several sensors, control methods, driving assistants, power train, etc. That represents the actual situation of most real producing companies with flexible series production.

Within limitations the customer here can also become the designer of its product. Those parts are produced on the already mentioned 3D printers and laser cutters. The product variety is with that almost unlimited.

The production is organised in a way that the catalogue options form the manufacturer and the customer individual part are produced simultaneously considering the individual production times especially for $3 \mathrm{D}$ printing.

Both material flows have to meet in time on work place three to be merged together.

\section{Automation and onotology}

In the smart factory intelligence is decentralised and linked together. That means every machine, device, sensor, even the product, needs to communicate open (UA-Community, 2013) with each other and finally also interact with each other. Therefore it requires a very specific communication concept and component standardisation within the factory but also beyond.

Figure 5 Automation concept of the smart factory of HTWG Constance (see online version for colours)

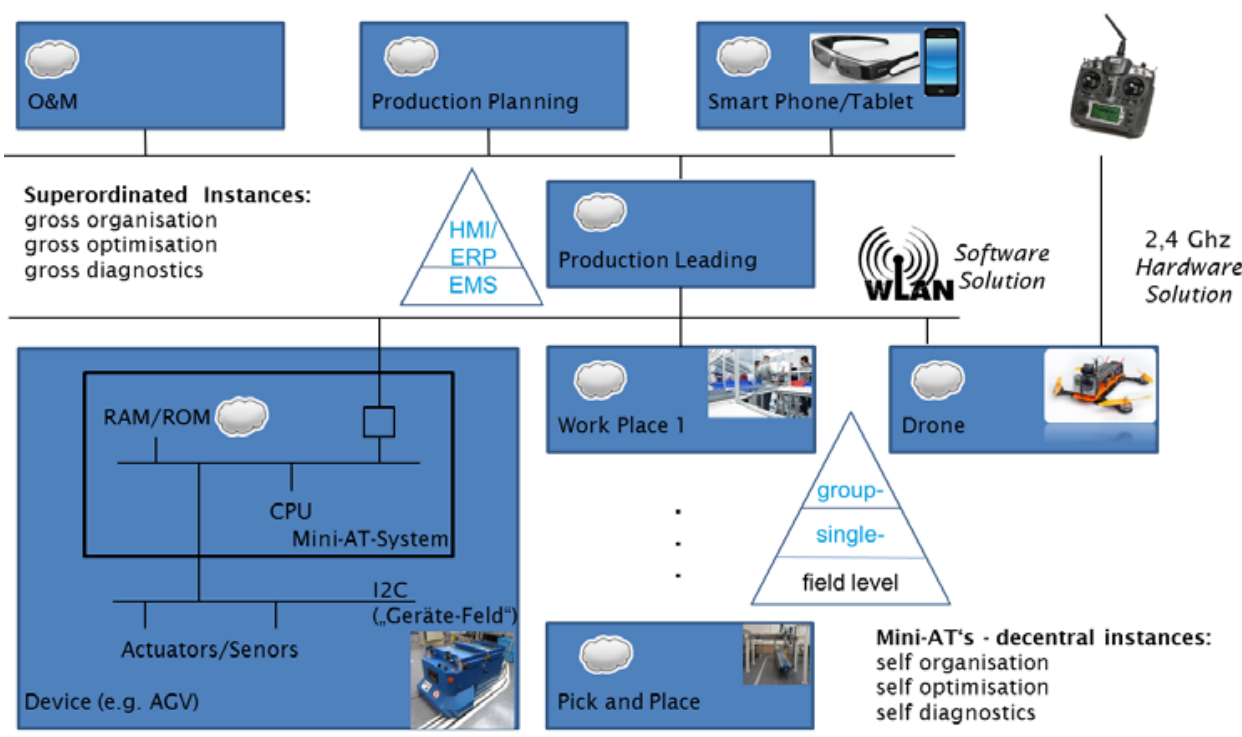

Figure 5 shows the automation concept of the smart factory. It is founded on massively standardised, small and open hard- and software together with an integrated graphical 
programming environment. The basic components are the same used in the cyber physical product.

On the first glance, the well introduced strongly hierarchic automation pyramid is dissolved by the network approach. That structure is a sociological well established organisation for responsibilities, authorities and more to realise a common will of an organisation.

With that network approach parts of that pyramid are decentralised and multiplied so that the system is build up from multiple small devices maintaining the hierarchical levels of the classic automation pyramid instead of one big automation system. The advantage of that concept is that all kind of part systems can be interconnected as long as their communication protocol is open.

Crucial is the ontology in the sense of organising data and its communication realising a common will of manufacturer and consumer.

In the concept, each device must be able to assess its own data and rules must be kept to organise communication to other devices, group clouds, factory cloud and an overlaying community cloud.

In that way, data transmission is organised in an efficient way helping maintaining real-time operation with standard protocols.

A benefit of CPS and the internet of things is to have access to all data every time with every network capable device such as a smartphone, tablet or laptop. Thereby, the operating system is irrelevant due to everything working browser-based. That benefit is used in the smart factory also.

To meet all requirements, there are several topics to take into account such as real-time capability, reliability/redundancy, data security and some more.

Therefore, the communication concept is based on a superordinated cloud(s) interconnecting all existing devices. The cloud collects and manages data, receives and plans orders. But there is not only a communication between the existing devices and the cloud but also among the devices themselves directly. The ability to communicate with each other in conjunction with certain intelligence should lead to self-optimising, self-configuring and self-diagnosis devices what finally enables a strong adaption of the products under conditions of a highly flexible production.

The communication inside the factory is based on Ethernet provided by LAN and WLAN. To achieve real-time capability in communication with every network capable device modified web sockets are used. A web socket is a TCP-based protocol providing full-duplex communication channels over a TCP connection. Once opened the web socket keeps the connection alive. So there is no more handshake and HTTP header transmission necessary and thus much more efficient for small messages than TCP itself.

In some particular cases, it is necessary to establish a point to point connection between two devices. For example, to control a robot directly or in case of error analysis. Therefore, every device is able to provide its own WI-FI.

\section{Didactics concept}

Imparting the techniques and possibilities of CPS is based on four pillars: instruction, self-study, community and application. 
For that several courses respectively course forms are offered to meet the different requirements of the several school forms or rather on the job training. The self-study part is increased accordingly.

Additionally, the smart factory is intended by its several industrial and public supporters to be open for the general public and provide a platform to become engaged with that topic.

To meet the requirements of the wide variety of users of the smart factory without overloading the university capacities massive open online courses (MOOCs) are implemented. With the help of an internet platform mainly short and free online courses are offered to give a high number of participants an introduction into different topics of the smart factory and CPS in general. That type of courses combine videos, readings und questions with communities where lecturers, students and everyone interested in that topic can communicate with each other.

Figure 6 Community cloud of the smart factory (see online version for colours)

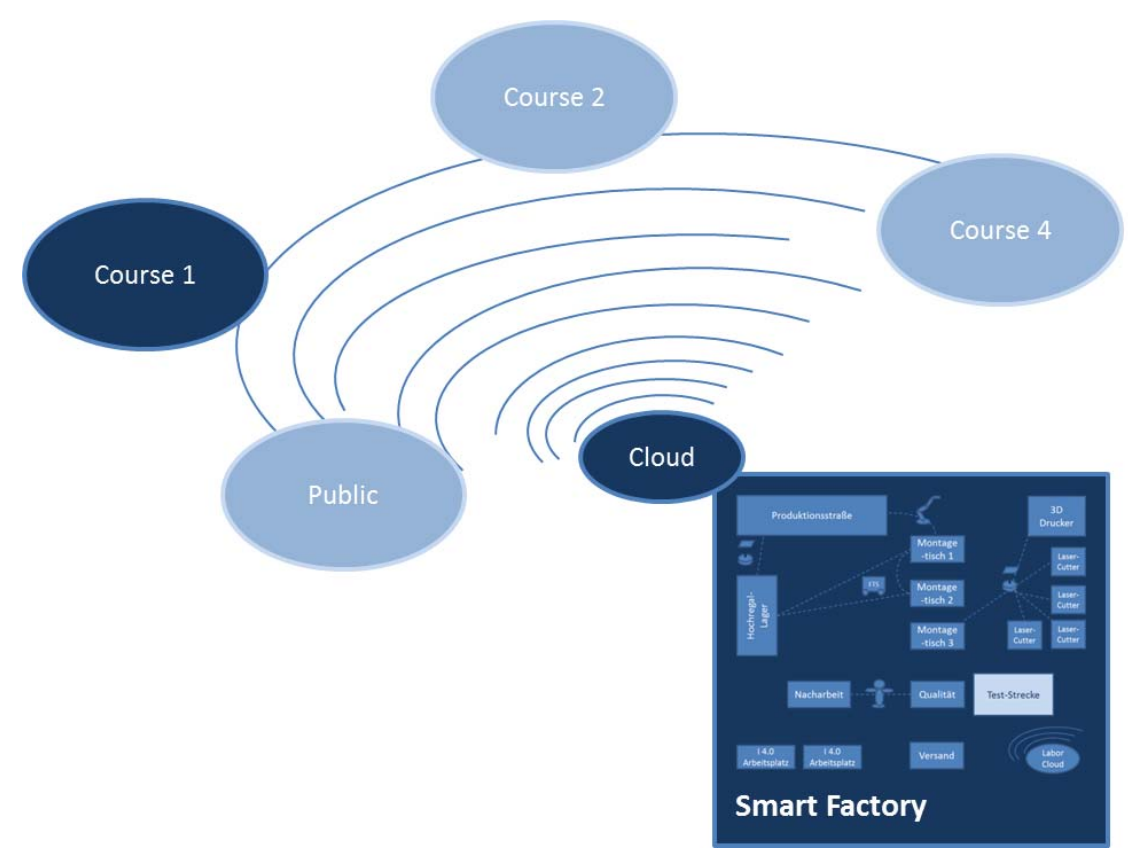

In the focus of the project are mainly short Mini-MOOCs with a length of a few minutes. That Mini-MOOCs are provided by a super ordinated community cloud, see Figure 6, connected to the above described factory cloud.

That community cloud is also intended for marketing for the courses liable for fees and for implementing new business models based on system dynamics analysis of data (Ihlenburg, 2010).

The courses base on the described principles. After succeeding in a Mini-MOOC, a corresponding course in a mentioned education facility is attended. There, a project oriented instruction in the form of seminars is offered. The participants have to prepare in self-study and apply their gained knowledge in the smart factory.

The course is completed by an according exam. 
The participants are required to participate interactively in the community cloud to familiarise with the topics, problems, application, etc. With that a way of self-check is provided as well.

\section{References}

ACATECH (2013) 'Umsetzungsempfehlungen für das zukunftsprojekt Industrie 4.0', in Kagermann, H., Wahlster, W. and Helbig, J. (Eds.): Abschlussbericht des Arbeitskreises Industrie 4.0, ACATECH, Munich.

Hesse, H., Kohn, A., Lehmann, J., Farazaneh, H. and Ihlenburg, D. (2014) 'Integration of semantic technologies for business process support in the automation industry', in Wahlster, W., Grallert, H-J., Wess, S., Friedrich, H. and Widenka, T. (HRSG.) (Eds.): Towards the Internet of Services, The Theseus Research Program, Springer Science + Business Media, Berlin.

Ihlenburg, D. (2010) 'Kundeninteraktion in der frühen phase des innovationsprozesses durch virtuelle interaktionsplattformen', erschienen Hofmann, J. (HG.) (Ed.): IT-Basiertes Innovationsmanagement, HMD, Heft 23, Praxis der Wirtschaftsinformatik, Berlin.

Rogers, E.M. (2003) Diffusion of Innovations, Free Press, New York.

Spath, D., Ganschar, O., Gerlach, S., Hämmerle, M., Krause, T. and Schlund, S. (2014) Produktionsarbeit der Zukunft - Industrie 4.0. Fraunhofer-Institut für Arbeitswirtschaft und Organisation IAO, Stuttgart.

Techconsult (2014) Business Performance Index, Expertenbericht Diskrete Fertigung, Kassel.

UA-Community, O. (2013) OPC Unified Architecture - Wegbereiter der Vierten Industriellen, Evolution, OPC Foundation, Verl.

VDI (2014) Industrie 4.0 - CPS-basierte Automation Forschungsbedarf Anhand Konkreter Fallbeispiele, Statusreport, Düsseldorf. 